\title{
Aspectos tecnológicos de sorgo-sacarino submetido à aplicação de maturadores químicos
}

\section{Technological aspects of sweet sorghum submitted to the application of ripeners chemicals}

\author{
Ronaldo da Silva VIANA ${ }^{1 *}$; André MAY ${ }^{2}$; Gustavo Pavan MATEUS ${ }^{3}$; Arthur Duarte RODRIGUES \\ $\mathrm{NETO}^{4}$; Paulo Renato Matos LOPES 5 \\ 1 Doutor em Agronomia; Faculdade de Ciências Agrárias e Tecnológicas de Dracena, Universidade Estadual Paulista - \\ UNESP, Rodovia Comandante João Ribeiro de Barros (SP 264), km 651,Bairro das Antas, Dracena-SP. CEP $17900-000$. \\ ronaldo@dracena.unesp.br \\ ${ }^{2}$ Doutor em Produção Vegetal; Empresa Agropecuária de Pesquisas - Embrapa- Jaguariúna. andre.may@embrapa.br \\ ${ }^{3}$ Doutor em Agronomia; Agência Paulista de Tecnologia em Agronegócio - Apta - Andradina. gpmateus@apta.sp.gov.br \\ ${ }^{4}$ Graduando em Agronomia; Faculdade de Ciências Agrárias e Tecnológicas de Dracena, Universidade Estadual Paulista - \\ UNESP.arthurodrigues@gmail.com \\ ${ }^{5}$ Doutor em Ciências Biológicas - Microbiologia Aplicada;Faculdade de Ciências Agrárias e Tecnológicas de Dracena, \\ Universidade Estadual Paulista - UNESP. plopes@dracena.unesp.br
}

Recebido em: 21-09-2016; Aceito em: 30-03-2017

\begin{abstract}
Resumo
O sorgo-sacarino apresentou um crescimento de interesse para cultivo em área de reformas de canaviais associadas a usinas sucroenergéticas em função da possibilidade de produção de etanol, na abertura da safra da cana-de-açúcar, sendo seu ponto de colheita normalmente identificado pela fenologia da planta, após o florescimento da cultura. A antecipação do momento de colheita e a possibilidade de acúmulo de açúcares no colmo, com a utilização de maturadores químicos, podem ser uma alternativa importante para a cultura, como ocorre com a cana-de-açúcar. Este trabalho teve como objetivo avaliar os aspectos tecnológicos do sorgo-sacarino quando aplicados diferentes maturadores químicos. O delineamento experimental utilizado foi o em blocos casualizados, em esquema fatorial 2x5, com 4 repetições, constituídos por: duas cultivares de sorgo-sacarino (BRS 508 e BRS 509) e cinco tratamentos com maturadores químicos ( $C$ - controle - ausência de maturador; $E$ - etefon $\left(0,7 \mathrm{~L} \mathrm{ha}^{-1}\right)$; $E T$ - etiltrinexapac $\left(0,8 \mathrm{~L} \mathrm{ha}^{-1}\right)$; $\mathrm{G}$ - glifosato $\left(0,4 \mathrm{~L} \mathrm{ha}^{-1}\right)$ e $\mathrm{S}$ - sulfometurom metil $\left(0,02 \mathrm{~kg} \mathrm{ha}^{-1}\right)$. Por ocasião da colheita do sorgo-sacarino, foram realizadas avaliações de sólidos solúveis (Brix do caldo, em \%); teor de sacarose no caldo - Pol (\%); Açúcares redutores (\%); Açúcares redutores totais (\%); Fibra (\%); Pureza (\%), e Açúcar total recuperável $\left(\mathrm{kg} \mathrm{t}^{-1}\right)$. A cultivar BRS 508 apresentou aumento na qualidade tecnológica quando utilizado sulfometurom metil $\left(0,02 \mathrm{~kg} \mathrm{ha}^{-1}\right)$, enquanto para a cultivar BRS 509, os ganhos ocorreram quando foi aplicado 0 etiltrinexapac $\left(0,8 \mathrm{~L} \mathrm{ha}^{-1}\right)$ como maturador.
\end{abstract}

Palavras-chave adicionais: estresse; maturação; reguladores vegetais, Sorghum bicolor.

\begin{abstract}
The sweet sorghum showed a growth of interest for cultivation in the area of sugarcane fields associated reforms sucroenergéticas plants due to ethanol production possibility at the opening of the harvest of sugarcane, and its point of harvest usually identified by phenology plant, after flowering of culture. The anticipation of the harvest time and the possibility of accumulation of sugars in the stem with the use of chemical ripeners can be an important alternative to the culture, as with sugarcane. This study aimed to evaluate the technological aspects of sweet sorghum when applied different ripeners chemicals. O experimental design was randomized blocks in a $2 \times 5$ factorial scheme, with four replications, consisting of: two cultivars of sweet sorghum (BRS 508 and BRS 509) and five treatments with chemical ripeners, C - control - absence of maturing; $S$ - sulfometurom methyl (0.02 kg ha-1); $E$ - ethephon (0.7 $\left.\mathrm{L} \mathrm{ha}^{-1}\right) ; \mathrm{G}$ - glyphosate $\left(0.4 \mathrm{~L} \mathrm{ha}^{-1}\right)$ and $\mathrm{E}$ - trinexapac-ethyl $\left(0.8 \mathrm{~L} \mathrm{~h}^{-1}\right)$ at the time of application, the products were mixed with $0.5 \%$ mineral oil for better adhesion on the sheet. During the harvest of sweet sorghum were made the following assessments: soluble solids Brix (\% juice); sucrose content of the juice - Pol (\% juice ); Reducing sugars; total reducing sugars ; Fiber (\%); Purity (\%) and total recoverable sugars (ATR $\mathrm{kg} \mathrm{t}^{-1}$ ). The cultivar BRS 508 showed an increase in technological quality when used sulfometurom methyl $\left(0.02 \mathrm{~kg} \mathrm{ha}^{-1}\right)$, while the BRS 509 , the gains occurred when applied trinexapac-ethyl $\left(0.8 \mathrm{~L} \mathrm{ha}^{-1}\right)$ as ripener.
\end{abstract}

Additional keywords: maturation, plant growth regulators, Sorghum bicolor; stress. 


\section{Introdução}

No Brasil, a matéria-prima utilizada com maior rendimento por área cultivada para a produção de etanol e açúcar é a cana-de-açúcar. Todavia, continuam as buscas por culturas que sirvam como matérias-primas renováveis e apresentem ciclo de desenvolvimento curto. Dentre essas culturas, uma que se destaca é o sorgo-sacarino (Viana et al., 2015).

O sorgo é uma espécie vegetal originária da África. No Brasil, consideram-se cinco tipos de sorgo importantes para o mercado, sendo eles: granífero, forrageiro, pastejo, sacarino e biomassa (May et al., 2011).

O sorgo-sacarino é uma cultura com potencial para a produção etanol, em função dos açúcares fermentescíveis presentes nos colmos das plantas (sacarose, glicose e frutose). Esta cultura apresenta grande potencial em razão de seu ciclo curto e das possibilidades de mecanização de todos os seus processos agrícolas (Sipos et al., 2009).

O sorgo-sacarino pode ser cultivado em áreas de reforma de canaviais, visando à colheita no período da entressafra da cana-de-açúcar, reduzindo a ociosidade da indústria em alguns dias (Durães, 2011).

A utilização de maturadores químicos na cultura da cana-de-açúcar é uma realidade cada vez mais frequente, pois eles facilitam o planejamento de colheita e aumentam a produtividade agroindustrial. Contudo, a utilização dos mesmos produtos recomendados para a cana-de-açúcar, em outras culturas, ainda demanda estudos mais aprofundados. No Brasil, têm sido utilizados, preferencialmente, os maturadores classificados como retardadores de crescimento (etefon e etiltrinexapac) e inibidores do crescimento (glifosato e sulfometurom metil), conforme retratam Viana et al. (2016).

Os maturadores químicos são substâncias sintéticas com ações similares aos hormônios das plantas. Assim, são produtos aplicados com a finalidade de antecipar o processo de maturação, promover melhorias na qualidade da matéria-prima, aperfeiçoar os resultados agroindustriais e econômicos e auxiliar no planejamento da safra, permitindo o indispensável manejo da cultura em seu moderno sistema de produção (Benedini \& Ricci-Júnior, 2009; Muhwiridzwa et al., 2016).

Esses reguladores vegetais alteram a morfologia e a fisiologia da planta, podendo levar a modificações qualitativas e quantitativas na produção agrícola, possibilitando incrementos do teor de sacarose, precocidade de maturação e aumento na produtividade (Caputo et al., 2007). Tais compostos favorecem o acúmulo mais uniforme de açúcares nos entrenós da região apical, que normalmente são imaturos. São aplicados com a finalidade de antecipar o processo de maturação, de promover melhorias na qualidade da matéria-prima a ser processada, de otimizar os resultados agroindustriais e econômicos, além de auxiliar no planejamento da safra em cana-de-açúcar (Viana et al., 2015)

Por outro lado, o uso de maturadores químicos na cultura do sorgo-sacarino é algo recente e carece de muitas pesquisas para conhecer melhor algumas implicações na qualidade da matéria-prima e aspectos tecnológicos da cultura.

Diante do exposto, o objetivo deste trabalho foi avaliar os aspectos tecnológicos do sorgo-sacarino quando aplicados diferentes maturadores químicos.

\section{Material e métodos}

O experimento foi instalado e conduzido no campo experimental pertencente à Agência Paulista de Tecnologia do Agronegócio (APTA), situada no município de Andradina-SP, com coordenadas geográficas $20^{\circ} 50^{\prime} 23^{\prime \prime S} / 51^{\circ} 20^{\prime} 37^{\prime \prime} \mathrm{W}$.

Por ocasião do preparo do solo, foi realizada sua correção e adubação, seguindo as recomendações técnicas, em função da análise do solo (Tabela 1), conforme Raij et al. (1996). A semeadura das cultivares de sorgo-sacarino BRS 508 e BRS 509 ocorreu no dia 10 de dezembro de 2015, utilizando 14 a 17 sementes por metro linear, espaçamento de 0,7 metro nas entrelinhas e profundidade de semeadura de 2 centímetros. Os tratamentos fitossanitários das plantas de sorgo ocorreram conforme as recomendações técnicas da cultura.

$O$ delineamento experimental utilizado foi o em blocos casualizados, em esquema fatorial $2 \times 5$, com quatro repetições, constituídos por: duas cultivares de sorgo-sacarino (BRS 508 e BRS 509) e cinco tratamentos com maturadores químicos ( $\mathrm{C}$ - controle - ausência de maturador; $E$ - etefon $\left(0,7 \mathrm{~L} \mathrm{ha}^{-1}\right)$; ET - etiltrinexapac $\left(0,8 \mathrm{~L} \mathrm{ha}^{-1}\right) ; \mathrm{G}$ - glifosato $\left(0,4 \mathrm{~L} \mathrm{ha}^{-1}\right)$ e $S$ - sulfometurom metil $\left(0,02 \mathrm{~kg} \mathrm{ha}^{-1}\right)$. No momento da aplicação, os produtos foram misturados com óleo mineral a 0,5\% para melhor adesão nas folhas . Cada parcela foi formada por cinco linhas espaçadas de $0,70 \mathrm{~m}$ entre si, com $5 \mathrm{~m}$ de comprimento, sendo as amostras colhidas nas linhas centrais.

As cultivares estudadas pertencem à Embrapa, caracterizadas com ciclo de 110 a 120 dias. O experimento foi instalado em 20 de fevereiro de 2016, quando foram aplicados os maturadores químicos no início da fase de florescimento das plantas, que ocorreu, aproximadamente, aos 70 dias após a semeadura. A aplicação de maturadores químicos foi realizada em uma lavoura sadia de sorgo-sacarino, utilizando um pulverizador costal pressurizado por $\mathrm{CO}_{2} \mathrm{e}$ barra de $4 \mathrm{~m}$ de comprimento, em formato de $\mathrm{T}$, com 8 pontas de jato plano AXI 11002, espaçadas em 0,5 m, possibilitando a aplicação simultânea nas linhas. A pressão utilizada foi de 40 libras pol-2 $^{-2}$, com volume de calda de $200 \mathrm{~L} \mathrm{ha}^{-1}$. A aplicação iniciou-se às $08 \mathrm{~h}$ e terminou às $11 \mathrm{~h}$ da manhã, período em que foi observada baixa ocorrência de ventos. A temperatura no momento da aplicação foi em torno de $25^{\circ} \mathrm{C}$, sem ocorrência de chuvas subsequentes. 
Tabela1 - Análise química do solo na profundidade de 0,00-0,20 e 0,20-0,40 m, antes da implantação do experimento. Andradina-SP, 2015. Soil chemical analysis at 0.00-0.20 and 0.20-0.40 m depth, before the implantation of the experiment. Andradina, São Paulo State - Brazil, 2015.

\begin{tabular}{|c|c|c|}
\hline & \multicolumn{2}{|c|}{ Profundidade $(m)$} \\
\hline & $0,00-0,20$ & $0,20-0,40$ \\
\hline $\mathrm{pH} \mathrm{CaCl}_{2}$ & 5,6 & 5,2 \\
\hline $\mathrm{MO} \mathrm{g} \mathrm{dm}-3$ & 13 & 9 \\
\hline $\mathrm{P}, \mathrm{mg} \mathrm{dm}^{-3}$ (resina) & 2 & 3 \\
\hline $\mathrm{K}, \mathrm{mmol}_{\mathrm{c}} \mathrm{dm}^{-3}$ (resina) & 2,7 & 2,5 \\
\hline $\mathrm{Ca}, \mathrm{mmol}_{\mathrm{c}} \mathrm{dm}^{-3}$ (resina) & 13 & 12 \\
\hline $\mathrm{Mg}, \mathrm{mmol}_{\mathrm{c}} \mathrm{dm}^{-3}$ (resina) & 7 & 7 \\
\hline $\mathrm{H}+\mathrm{Al}, \mathrm{mmol}_{\mathrm{c}} \mathrm{dm}^{-3}$ & 18 & 20 \\
\hline $\mathrm{Al}, \mathrm{mmol}_{\mathrm{c}} \mathrm{dm}^{-3}$ & 0 & 0 \\
\hline Soma de bases, $\mathrm{mmol}_{\mathrm{c}} \mathrm{dm}^{-3}$ & 23 & 22 \\
\hline CTC, $\mathrm{mmolc}_{\mathrm{c}} \mathrm{dm}^{-3}$ & 41 & 42 \\
\hline Saturação por bases, \% & 56 & 52 \\
\hline Saturação Al, \% & 0 & 0 \\
\hline $\mathrm{S}\left(\mathrm{SO}_{4}^{-2}\right), \mathrm{mg} \mathrm{dm}^{-3}$ & 8 & 11 \\
\hline $\mathrm{Cu}, \mathrm{mg} \mathrm{dm}^{-3}$ (DTPA) & 0,6 & 0,8 \\
\hline $\mathrm{Fe}, \mathrm{mg} \mathrm{dm}^{-3}$ (DTPA) & 15 & 11 \\
\hline $\mathrm{Zn}, \mathrm{mg} \mathrm{dm}^{-3}$ (DTPA) & 0,4 & 0,4 \\
\hline $\mathrm{Mn}, \mathrm{mg} \mathrm{dm}^{-3}$ (DTPA) & 14,0 & 8,8 \\
\hline $\mathrm{B}, \mathrm{mg} \mathrm{dm}^{-3}$ (Água quente) & 0,15 & 0,28 \\
\hline Argila, $\mathrm{g} \mathrm{kg}^{-1}$ & 113 & 137 \\
\hline Silte, $\mathrm{g} \mathrm{kg}^{-1}$ & 37 & 33 \\
\hline Areia total, $\mathrm{g} \mathrm{kg}^{-1}$ & 850 & 830 \\
\hline Areia grossa, $\mathrm{g} \mathrm{kg}^{-1}$ & 150 & 120 \\
\hline Areia fina, $\mathrm{g} \mathrm{kg}^{-1}$ & 700 & 710 \\
\hline
\end{tabular}

MO: matéria orgânica; CTC: capacidade de troca de cátions. MO: organic matter; CTC: cation exchange capacity

Aos 40 dias após a aplicação dos maturadores químicos, foi realizada a colheita das plantas, visando à realização das avaliações tecnológicas do caldo das plantas. Nesse momento, foram colhidos 2 metros lineares de cada parcela, sendo retiradas as folhas das plantas e os colmos despontados na altura da gema apical, sendo conduzidos ao laboratório da Usina Caeté para análise das seguintes características do caldo extraído dos colmos de sorgo-sacarino após prensagem em prensa hidráulica: sólidos solúveis (Brix, em \%); teor de sacarose no caldo - Pol (\%); Açúcares redutores (AR, em \%); Açúcares redutores totais (ART, em \%); Fibra (\%); Pureza (\%), e Açúcar total recuperável (ATR, em kg/tonelada de colmo), segundo os métodos de determinações químico-tecnológicas descritos pelo Consecana (2006).

As variáveis foram submetidas à análise de variância, pelo teste $F(p<0,05)$, e suas médias, comparadas pelo teste de Tukey, a $5 \%$ probabilidade, sendo utilizado o programa Assistat 7.6 Beta (Silva \& Azevedo, 2002).

\section{Resultados e discussão}

$\mathrm{Na}$ Tabela 2, estão apresentados os valores médios dos parâmetros tecnológicos de Brix; Pol;
Açúcares redutores (AR), e Açúcares redutores totais (ART) das cultivares de sorgo-sacarino, submetidas à aplicação de maturadores químicos. Houve diferença significativa isolada para a maioria dos fatores, em todas as características tecnológicas estudadas. Houve também interações significativas entre os fatores estudados.

Dessa forma, na Tabela 3, são apresentados os valores médios relativos ao desdobramento da interação significativa entre as duas cultivares de sorgosacarino submetidas à aplicação de maturadores químicos, para a característica tecnológica Brix caldo.

Para a característica Brix do caldo, houve diferença significativa entre as cultivares de sorgo-sacarino, sendo que a cultivar BRS 508 apresentou os maiores valores, quando foram aplicados os maturadores sulfometurom metil e etefon. $\mathrm{O}$ tratamento com maturador químico sulfometurom metil exibiu os melhores resultados na cultivar BRS 508, enquanto o tratamento com maturador químico etiltrinexapac apresentou melhores valores de Brix do caldo para a cultivar BRS 509 (Tabela 3). Estes dados corroboram Viana et al. (2016), que verificaram ganho nas características tecnológicas do Brix do caldo quando foram aplicados os maturadores químicos sulfometuronmethyl e etephon em sorgo-sacarino. 
Tabela 2 - Valores médios dos parâmetros tecnológicos de Brix; Pol; Açúcares redutores (AR), e Açúcares redutores totais (ART) de duas cultivares de sorgo-sacarino submetido à aplicação de maturadores químicos, Andradina-SP, 2015. Mean values of the technological parameters of Brix; Pol; Reducing sugars (AR) and total reducing sugars (ART) of two cultivars of sweet sorghum submitted to the application of chemical ripeners, Andradina, São Paulo State - Brazil, 2015.

\begin{tabular}{lcccc}
\hline Causas da variação & $\begin{array}{c}\text { Brix } \\
(\%)\end{array}$ & $\begin{array}{c}\text { Pol } \\
(\%)\end{array}$ & $\begin{array}{c}\text { AR } \\
(\%)\end{array}$ & $\begin{array}{c}\text { ART } \\
\left(\mathrm{kg} \mathrm{t}^{-1}\right)\end{array}$ \\
\hline Cultivares (C) & $23,81 \mathrm{a}$ & $19,52 \mathrm{a}$ & $0,82 \mathrm{a}$ & $151,06 \mathrm{a}$ \\
BRS 508 & $23,30 \mathrm{~b}$ & $19,17 \mathrm{~b}$ & $0,81 \mathrm{a}$ & $151,77 \mathrm{a}$ \\
BRS 509 & 0,20 & 0,30 & 0,02 & 0,91 \\
\hline DMS & $27,09^{* *}$ & $19,20^{* *}$ & $0,58 \mathrm{~ns}$ & $2,58 \mathrm{~ns}$ \\
\hline F(C) & & & & \\
\hline Maturadores (M) & $22,94 \mathrm{~b}$ & $19,08 \mathrm{~b}$ & $0,79 \mathrm{~b}$ & $148,60 \mathrm{c}$ \\
C & $23,99 \mathrm{a}$ & $19,96 \mathrm{a}$ & $0,78 \mathrm{~b}$ & $157,98 \mathrm{a}$ \\
E & $24,14 \mathrm{a}$ & $20,26 \mathrm{a}$ & $0,75 \mathrm{~b}$ & $157,15 \mathrm{a}$ \\
ET & $22,27 \mathrm{c}$ & $18,05 \mathrm{c}$ & $0,86 \mathrm{a}$ & $139,54 \mathrm{~d}$ \\
G & $24,44 \mathrm{a}$ & $19,37 \mathrm{~b}$ & $0,91 \mathrm{a}$ & $153,80 \mathrm{~b}$ \\
S & 0,45 & 0,36 & 0,06 & 2,06 \\
\hline DMS & $69,18^{* *}$ & $93,50^{* *}$ & $17,43^{\star *}$ & $231,91^{* *}$ \\
\hline F(M) & 1,32 & 1,31 & 5,56 & 0,93 \\
\hline CV\% & $69,58^{* *}$ & $24,12^{* *}$ & $32,82^{* *}$ & $49,98^{* *}$ \\
\hline C x M & $(P 0,01) ;$ & & \\
\hline
\end{tabular}

${ }^{*}$ Significativo ao nível de $1 \%$ de probabilidade $(p<0,01)$; ${ }^{*}$ Significativo ao nível de $5 \%$ de probabilidade $(0,01=<p<0,05)$; ns - Não significativo ( $p>=0,05)$; Teste de Tukey a $5 \%$ de probabilidade; daCV - Coeficiente de variação; $C$ - Cultivares ; M Maturadores; E - Época; DMS - Diferença mínima significativa; C - Controle - ausência de maturador; E - Etefon $\left(0,7 \mathrm{~L} \mathrm{ha}^{-1}\right)$; ET - Etiltrinexapac $\left(0,8 \mathrm{~L} \mathrm{ha}^{-1}\right) ; \mathrm{G}$ - Glifosato $\left(0,4 \mathrm{~L} \mathrm{ha}^{-1}\right)$ e S - Sulfometurom metil $\quad\left(0,02 \mathrm{~kg} \mathrm{ha}^{-1}\right)$

${ }^{*}$ Significant at the $1 \%$ probability level $(p<0.01)$; ${ }^{*}$ Significant at the $5 \%$ probability level $(0.01=<p<0.05)$; Ns - Not significant $(p>=0.05)$; Tukey test $5 \%$ probability; $C V$ - Coefficient of variation; $C$ - Cultivars; $M$ - Maturadores; $E$ - Season; DMS Minimum significant difference; $C$ - Control - absence of maturer; $E$ - Ethephon $\left(0.7 L\right.$ ha $\left.^{-1}\right)$; $E T$ - Ethyl- $\quad$-trinexapac $(0.8$ $\left.L \mathrm{ha}^{-1}\right)$; G-Glyphosate $\left(0.4 \mathrm{~L} \mathrm{ha}^{-1}\right)$ and S - Sulfometurom methyl $\left(0.02 \mathrm{~kg} \mathrm{ha}^{-1}\right)$.

Tabela 3 - Valores médios relativos ao desdobramento da interação significativa entre as duas cultivares de sorgo-sacarino submetidas à aplicação de maturadores químicos, para a característica tecnológica Brix caldo, Andradina-SP, 2015. Mean values related to the split of the significant interaction between the two cultivars of sweet sorghum submitted to the application of chemical ripeners, for the technological feature Brix broth, Andradina, São Paulo State - Brazil, 2015.

\begin{tabular}{lccccc}
\hline \multirow{2}{*}{ Cultivares } & \multicolumn{5}{c}{$\begin{array}{c}\text { Brix } \\
(\%)\end{array}$} \\
\cline { 2 - 6 } & $\mathrm{C}$ & $\mathrm{E}$ & $\mathrm{ET}$ & $\mathrm{G}$ & $\mathrm{S}$ \\
\hline BRS 508 & $23.50 \mathrm{aC}$ & $24.42 \mathrm{aB}$ & $22.85 \mathrm{bD}$ & $22.68 \mathrm{aD}$ & $25.60 \mathrm{aA}$ \\
BRS 509 & $22.30 \mathrm{bC}$ & $23.55 \mathrm{bB}$ & $25.42 \mathrm{aA}$ & $21.86 \mathrm{bC}$ & $23.28 \mathrm{bB}$ \\
\hline & DMS coluna: 0.44 & & DMS linha: 0.64 & & \\
\hline
\end{tabular}

Letras minúsculas comparam médias na coluna; Letras maiúsculas comparam médias na linha; Médias seguidas de letras iguais não diferem entre si, pelo teste de Tukey, a $5 \%$ de probabilidade, dentro do mesmo fator; DMS - Diferença mínima significativa; C - Controle - ausência de maturador; $\mathrm{E}$ - Etefon $\left(0,7 \mathrm{~L} \mathrm{ha}^{-1}\right)$; $\mathrm{ET}$ - Etiltrinexapac $\left(0,8 \mathrm{~L}\right.$ ha $\left.{ }^{-1}\right) ; \mathrm{G}$ - Glifosato $(0,4 \mathrm{~L}$ ha $\left.^{-1}\right)$ e S - Sulfometurom metil $\left(0,02 \mathrm{~kg} \mathrm{ha}^{-1}\right)$.

Lowercase letters compare averages in the column; Capital letters compare averages in the line; Averages followed by equal letters do not differ from each other by the Tukey test at $5 \%$ probability within the same factor; MS - Minimum significant difference; $C$ - Control - absence of maturer; $E$ - Ethephon ( $\left.0.7 \mathrm{~L} \mathrm{ha}^{-1}\right)$; $E T$ - Ethyl-trinexapac $(0.8 \mathrm{~L} \mathrm{ha-1})$; G - Glyphosate $(0.4 \mathrm{~L}$ $\left.\mathrm{ha}^{-1}\right)$ and $S$ - Sulfometurom methyl $\left(0.02 \mathrm{~kg} \mathrm{ha}^{-1}\right)$.

Caputo et al. (2008) relataram que o emprego de maturadores químicos destaca-se como uma ferramenta importante, pois são produtos aplicados com a finalidade de antecipar o processo de maturação, promovendo melhorias na qualidade da matéria-prima e auxiliando no planejamento da safra. $O$ mesmo pode ser observado neste trabalho, já que o uso de maturadores químicos promoveu melhorias na qualidade 
tecnológica do sorgo-sacarino. Resultados semelhante foram observados por Viana et al. (2015), na cultura de cana-de-açúcar, quando estudaram o efeito do uso de glyphosate e sulfometuron-methyl, sendo capazes de induzir o aumento de sacarose em todas as secções do colmo da cana-de-açúcar, em decorrência da aplicação dos produtos químicos.

Silva et al. (2007) e Silva et al. (2010), trabaIhando com cana-de-açúcar, também observaram que a aplicação de sulfometuron-metil ou etefon melhora a qualidade tecnológica dos colmos das plantas. De acordo com Prasad et al. (2007), o estágio ideal de colheita da cana-de-açúcar ocorre quando o caldo apresenta entre 15,5 e $16,5^{\circ}$ Brix, sendo este grau importante para obtenção de um caldo com alta qualidade de fermentação, com consequente maximização na produção de etanol por hectare. Já, outros autores aconselham médias mais altas de Brix para a colheita (Almodares \& Hadi, 2009).

$\mathrm{Na}$ Tabela 4, são apresentados os valores médios relativos ao desdobramento da interação significativa entre as duas cultivares de sorgo-sacarino submetidas à aplicação de maturadores químicos, para a característica tecnológica Pol do caldo.

Tabela 4 - Valores médios relativos ao desdobramento da interação significativa entre as duas cultivares de sorgo-sacarino submetidas à aplicação de maturadores químicos, para a característica tecnológica Pol caldo, Andradina-SP, 2015. Mean values for the split of the significant interaction between the two cultivars of sweet sorghum submitted to the application of chemical ripeners, for the technological characteristic Pol., Andradina, São Paulo State - Brazil, 2015.

\begin{tabular}{lccccc}
\hline \multirow{2}{*}{ Cultivares } & \multicolumn{7}{c}{$\begin{array}{c}\text { Pol } \\
(\%)\end{array}$} \\
\cline { 2 - 6 } & $\mathrm{C}$ & $\mathrm{E}$ & $\mathrm{ET}$ & $\mathrm{G}$ & $\mathrm{S}$ \\
\hline BRS 508 & $19.70 \mathrm{aA}$ & $20.20 \mathrm{aA}$ & $20.14 \mathrm{aA}$ & $17.64 \mathrm{bB}$ & $19.93 \mathrm{aA}$ \\
BRS 509 & $18.47 \mathrm{bC}$ & $19.73 \mathrm{bB}$ & $20.39 \mathrm{aA}$ & $18.45 \mathrm{aC}$ & $18.82 \mathrm{bC}$ \\
\hline \multicolumn{7}{c}{ DMS coluna: 0,36} & & DMS linha: 0,52 & & \\
\hline
\end{tabular}

Letras minúsculas comparam médias na coluna; Letras maiúsculas comparam médias na linha; Médias seguidas de letras iguais não diferem entre si, pelo teste de Tukey, a $5 \%$ de probabilidade, dentro do mesmo fator; DMS - Diferença mínima significativa; C - Controle - ausência de maturador; $\mathrm{E}$ - Etefon $\left(0,7 \mathrm{~L} \mathrm{ha}^{-1}\right)$; $\mathrm{ET}$ - Etiltrinexapac $\left(0,8 \mathrm{~L} \mathrm{ha}^{-1}\right) ; \mathrm{G}-\mathrm{Glifosato}(0,4 \mathrm{~L}$ $\left.\mathrm{ha}^{-1}\right)$ e S - Sulfometurom metil $\left(0,02 \mathrm{~kg} \mathrm{ha}^{-1}\right)$.

Lowercase letters compare averages in the column; Capital letters compare averages in the line; Averages followed by equal letters do not differ from each other by the Tukey test at $5 \%$ probability within the same factor; DMS - Minimum significant difference; $C$ - Control - absence of maturer; $E$ - Ethephon (0.7 L ha $\left.{ }^{-1}\right) ; E T$ - Ethyl-trinexapac (0.8 $L$ ha $\left.^{-1}\right)$; $G$ - Glyphosate (0.4 L $\mathrm{ha}^{-1}$ ) and S - Sulfometurom methyl (0.02 $\left.\mathrm{kg} \mathrm{ha}^{-1}\right)$.

Para a característica Pol do caldo, houve diferença significativa entre as cultivares de sorgo-sacarino, sendo que a cultivar BRS 508 apresentou os melhores valores nos tratamentos com a aplicação de etefon e sulfometurom metil. Já a BRS 509 apresentou melhores valores quando foi aplicado o maturador glifosato (Tabela 4). Estes dados corroboram Viana et al. (2016), trabalhando com sorgo-sacarino, que verificaram nas características tecnológicas analisadas, que os maturadores químicos sulfometuron-methyl e etephon, nas doses de $5 \mathrm{~g} \mathrm{ha}^{-1}$ e $0,33 \mathrm{~L} \mathrm{ha}^{-1}$, respectivamente, promoveram os melhores resultados.

Benedini \& Ricci-Júnior (2009) relataram que a eficiência agronômica dos maturadores depende da época de aplicação, da condição climática e da característica genética da variedade em cana-de-açúcar, quando cultivadas nas condições em que a maturação não é favorecida.

Leite et al. (2009a; 2009c) verificaram que a aplicação de maturadores químicos em cana-de-açúcar pode explorar o potencial genético das variedades quanto ao acúmulo de sacarose, melhorando a qualidade da matéria-prima.

Os valores médios relativos ao desdobramento da interação significativa entre as duas cultivares de sorgo-sacarino, submetidas à aplicação de maturado- res químicos, para a característica tecnológica açúcares redutores, são apresentados na Tabela 5.

Para a característica açúcares redutores (AR), houve diferença significativa entre as cultivares de sorgo-sacarino, sendo que a cultivar BRS 508 apresentou maiores valores nos tratamentos com glifosato e sulfometurom metil, em relação à cultivar BRS 509, enquanto a cultivar BRS 509 apresentou os maiores valores de AR quando aplicado o maturador etiltrinexapac (Tabela 5). Algumas cultivares de sorgo podem apresentar sensibilidade ao uso de maturadores, refletindo diretamente no conteúdo dos açúcares redutores que, com o aumento na planta, favorecem o crescimento vegetativo e afetam diretamente a pureza do caldo, refletindo em menor eficiência na recuperação da sacarose pela indústria. Por outro lado, a redução desses carboidratos melhora a qualidade tecnológica do caldo, favorecendo a maturação da cana-de-açúcar, conforme esclareceram Leite et al. (2009a). Conforme elucidaram Gomide et al. (2008), esse fato pode ter ocorrido em decorrência do uso de maturadores químicos, já que auxiliam na manutenção e na antecipação do ganho de sacarose, com consequente diminuição dos açúcares redutores.

Meschede et al. (2011) relataram que o sulfumeturon-methyl, em doses elevadas, pode interferir na 
fotossíntese, devido à diminuição dos teores de clorofila e carotenoides nas folhas. Os autores ressaltam as análises de clorofila e, principalmente, de carotenoides, sendo essas alterações um bom indicador para determinar os efeitos de fitotoxidade desses produtos na planta cultivada.
Os valores médios relativos ao desdobramento da interação significativa entre as duas cultivares de sorgo-sacarino submetidas à aplicação de maturadores químicos, para a característica tecnológica açúcares redutores totais, são apresentados na Tabela 6 .

Tabela 5 - Valores médios relativos ao desdobramento da interação significativa entre as duas cultivares de sorgo-sacarino submetidas à aplicação de maturadores químicos, para a característica tecnológica Açúcares redutores (AR), Andradina-SP, 2015. Mean values for the split of the significant interaction between the two cultivars of sweet sorghum submitted to the application of chemical ripeners, for the technological characteristic Reducing sugars (AR), Andradina, São Paulo State - Brazil, 2015.

\begin{tabular}{lccccc}
\hline \multirow{2}{*}{ Cultivares } & \multicolumn{5}{c}{$\begin{array}{c}\text { AR } \\
(\%)\end{array}$} \\
\cline { 2 - 6 } & $\mathrm{C}$ & $\mathrm{E}$ & $\mathrm{ET}$ & $\mathrm{G}$ & $\mathrm{S}$ \\
\hline BRS 508 & $0.77 \mathrm{aB}$ & $0.80 \mathrm{aB}$ & $0.61 \mathrm{bC}$ & $0.97 \mathrm{aA}$ & $0.97 \mathrm{aA}$ \\
BRS 509 & $0.81 \mathrm{aAB}$ & $0.77 \mathrm{aB}$ & $0.88 \mathrm{aA}$ & $0.75 \mathrm{bB}$ & $0.86 \mathrm{bA}$ \\
\hline & DMS coluna: 0.06 & & DMS linha: 0.09 & & \\
\hline
\end{tabular}

Letras minúsculas comparam médias na coluna; Letras maiúsculas comparam médias na linha; Letras iguais não diferem entre si, pelo teste de Tukey, a 5\% de probabilidade, dentro do mesmo fator; MS - Diferença mínima significativa; C - Controle - ausência de maturador; $\mathrm{E}$ - Etefon $\left(0,7 \mathrm{~L} \mathrm{ha}^{-1}\right)$; ET - Etiltrinexapac $\left(0,8 \mathrm{~L} \mathrm{ha}^{-1}\right) ; \mathrm{G}$ - Glifosato $(0,4 \mathrm{~L}$ ha-1) e S - Sulfometurom metil $\left(0,02 \mathrm{~kg} \mathrm{ha}^{-1}\right)$. Lowercase letters compare averages in the column; Capital letters compare averages in the line; Equal letters do not differ from each other by the Tukey test at 5\% probability within the same factor; DMS - Minimum significant difference; C - Control - absence of maturer; $E$ - Ethephon (0.7 L ha-1); ET - Ethyl-trinexapac (0.8 L ha $\left.{ }^{-1}\right)$; G - Glyphosate (0.4 L $\left.\mathrm{ha}^{-1}\right)$ and $S$ - Sulfometurom methyl $\left(0.02 \mathrm{~kg} \mathrm{ha}^{-1}\right)$.

Tabela 6 - Valores médios relativos ao desdobramento da interação significativa entre as duas cultivares de sorgo-sacarino submetidas à aplicação de maturadores químicos, para a característica tecnológica Açúcares redutores totais (ART), Andradina-SP, 2015. Mean values related to the split of the significant interaction between the two cultivars of sweet sorghum submitted to the application of chemical ripeners, for the technological characteristic Total reducing sugars (ART), Andradina, São Paulo State - Brazil, 2015.

\begin{tabular}{cccccc}
\hline \multirow{2}{*}{ Cultivares } & \multicolumn{7}{c}{$\begin{array}{c}\text { ART } \\
(\%)\end{array}$} \\
\cline { 2 - 6 } & $\mathrm{C}$ & $\mathrm{E}$ & $\mathrm{ET}$ & $\mathrm{G}$ & $\mathrm{S}$ \\
\hline BRS 508 & $148.18 \mathrm{aC}$ & $157.76 \mathrm{aA}$ & $151.14 \mathrm{bB}$ & $140.96 \mathrm{aD}$ & $157.25 \mathrm{aA}$ \\
BRS 509 & $149.02 \mathrm{Ac}$ & $158.21 \mathrm{aB}$ & $163.17 \mathrm{aA}$ & $138.13 \mathrm{bD}$ & $150.35 \mathrm{bC}$ \\
\hline \multicolumn{7}{c}{ DMS coluna: 2.04} & \multicolumn{7}{c}{ DMS linha: 2.91} \\
\hline
\end{tabular}

Letras minúsculas comparam médias na coluna; Letras maiúsculas comparam médias na linha; Médias seguidas de letras iguais não diferem entre si, pelo teste de Tukey, a $5 \%$ de probabilidade, dentro do mesmo fator; DMS - Diferença mínima significativa; $\mathrm{C}$ - Controle - ausência de maturador; $\mathrm{E}$ - Etefon $\left(0,7 \mathrm{~L} \mathrm{ha}^{-1}\right) ; \mathrm{ET}$ - Etiltrinexapac $\left(0,8 \mathrm{~L} \mathrm{ha} \mathrm{C}^{-1}\right) ; \mathrm{G}$ - Glifosato $(0,4 \mathrm{~L}$ $\left.\mathrm{ha}^{-1}\right)$ e S - Sulfometurom metil $\left(0,02 \mathrm{~kg} \mathrm{ha}^{-1}\right)$. Lowercase letters compare averages in the column; Capital letters compare averages in the line; Averages followed by equal letters do not differ from each other by the Tukey test at 5\% probability within the same factor; MS - Minimum significant difference; $C$ - Control - absence of maturer; $E$ - Ethephon (0.7 L ha $\left.{ }^{-1}\right) ; E T$ - Ethyltrinexapac $\left(0.8 \mathrm{~L} \mathrm{ha}^{-1}\right) ; \mathrm{G}$ - Glyphosate $\left(0.4 \mathrm{~L} \mathrm{ha}^{-1}\right)$ and S - Sulfometurom methyl $\left(0.02 \mathrm{~kg} \mathrm{ha}^{-1}\right)$.

Houve diferença significativa entre as cultivares de sorgo-sacarino, sendo que a cultivar BRS 509 apresentou maiores valores no tratamento com etiltrinexapac, enquanto a cultivar BRS 508 apresentou os maiores valores de ART quando submetidos à aplicação dos maturadores químicos etefon sulfometurom metil (Tabela 6). O uso de maturadores na indução artificial da maturação, em cana-de-açúcar é bem comum e paralisa o crescimento da altura da planta e modifica a partição dos fotoassimilados, deslocandoos para o acúmulo de sacarose, conforme explica Meschede et al. (2011). Silva et al. (2010) relataram que o fitorregulador sulfometuron methyl, quando apli- cado em diferentes variedades de cana-de-açúcar, possibilita a melhoria da qualidade tecnológica, promovendo resposta significativa com relação aos ganhos de Pol, aumentos da Pureza e redução no teor de ácidos orgânicos do caldo.

$\mathrm{Na}$ Tabela 7, foram apresentados os valores médios dos parâmetros tecnológicos de Pureza, Fibra e açúcares totais recuperáveis (ATR) de duas cultivares de sorgo-sacarino, submetidas à aplicação de maturadores químicos. Houve diferença significativa entre a maioria dos fatores isoladamente para as características tecnológicas avaliadas, assim como houve interações significativas entre os fatores. 
Tabela 7 - Valores médios dos parâmetros produtivo e tecnológicos de Pureza Fibra e Açúcares totais recuperáveis (ATR) de duas cultivares de sorgo-sacarino submetidas à aplicação de maturadores químicos, AndradinaSP, 2015. Average values of the productive and technological parameters of Purity; Fibra and total recoverable sugars (ATR) of two cultivars of sweet sorghum submitted to the application of chemical ripeners, Andradina, São Paulo State - Brazil, 2015.

\begin{tabular}{lccc}
\hline Causas da variação & $\begin{array}{c}\text { Pureza } \\
(\%)\end{array}$ & $\begin{array}{c}\text { Fibra } \\
(\%)\end{array}$ & $\begin{array}{c}\text { ATR } \\
(\mathrm{kg} / \mathrm{t})\end{array}$ \\
\hline Cultivares (C) & & & \\
BRS 508 & $82,11 \mathrm{a}$ & $21,91 \mathrm{a}$ & $138,22 \mathrm{a}$ \\
BRS 509 & $82,35 \mathrm{a}$ & $20,78 \mathrm{~b}$ & $138,87 \mathrm{a}$ \\
\hline DMS & 0,87 & 0,19 & 079 \\
\hline F(C) & $0,31 \mathrm{~ns}$ & $145,87^{* *}$ & $2,83 \mathrm{~ns}$ \\
\hline Maturadores (M) & & & \\
C & $83,17 \mathrm{a}$ & $21,52 \mathrm{~b}$ & $135,97 \mathrm{c}$ \\
E & $83,27 \mathrm{a}$ & $20,60 \mathrm{c}$ & $144,56 \mathrm{a}$ \\
ET & $84,29 \mathrm{a}$ & $21,58 \mathrm{~b}$ & $143,79 \mathrm{a}$ \\
G & $81,09 \mathrm{~b}$ & $22,20 \mathrm{a}$ & $127,68 \mathrm{~d}$ \\
C & $79,34 \mathrm{~b}$ & $20,82 \mathrm{c}$ & $140,73 \mathrm{~b}$ \\
\hline DMS & 1,96 & 0,43 & 1,78 \\
\hline F(M) & $17,45^{* *}$ & $37,96^{* *}$ & $257,68^{* *}$ \\
\hline CV\% & 1,64 & 1,38 & 0,88 \\
\hline C x M & $33,43^{* *}$ & $128,92^{* *}$ & $55,62^{* *}$ \\
\hline
\end{tabular}

${ }^{* *}$ Significativo ao nível de $1 \%$ de probabilidade $(p<0,01)$; ${ }^{*}$ Significativo ao nível de $5 \%$ de probabilidade $(0,01=<p<0,05)$; ns - não significativo ( $p>=0,05)$; CV - coeficiente de variação; DMS - Diferença mínima significativa; $C$ - Controle ausência de maturador; E - Etefon $\left(0,7 \mathrm{~L} \mathrm{ha}^{-1}\right)$; ET - Etiltrinexapac $\left(0,8 \mathrm{~L} \mathrm{ha}^{-1}\right)$; $\mathrm{G}$ - Glifosato $\left(0,4 \mathrm{~L}^{-1} \mathrm{ha}^{-1}\right)$ e $\mathrm{S}$ Sulfometurom metil $\left(0,02 \mathrm{~kg} \mathrm{ha}^{-1}\right) .{ }^{* *}$ Significant at the $1 \%$ probability level $(p<0.01) ;{ }^{*}$ Significant at the $5 \%$ probability level $(0.01=<p<0.05) ; \mathrm{Ns}$ - Not significant ( $p>=0.05) ; \mathrm{CV}$ - coefficient of variation; MS - Minimum significant difference; $C$ - Control - absence of maturer; $E$ - Ethephon $\left(0.7 L h^{-1}\right)$; $E T$ - Ethyl-trinexapac $\left(0.8 L h^{-1}\right) ; G$ - Glyphosate $\left(0.4 L \mathrm{ha}^{-1}\right)$ and $\mathrm{S}$ - Sulfometurom methyl $\left(0.02 \mathrm{~kg} \mathrm{ha}^{-1}\right)$.

$\mathrm{Na}$ Tabela 8, são apresentados os valores médios relativos ao desdobramento da interação significativa entre as duas cultivares de sorgo-sacarino

submetidas à aplicação de maturadores químicos, para a característica tecnológica Pureza do caldo.

Tabela 8 - Valores médios relativos ao desdobramento da interação significativa entre as duas cultivares de sorgo-sacarino submetidas à aplicação de maturadores químicos, para a característica tecnológica Pureza, Andradina-SP, 2015. Mean values related to the split of the significant interaction between the two sweet sorghum cultivars submitted to the application of chemical ripeners, for the technological characteristic Pureza, Andradina, São Paulo State - Brazil, 2015.

\begin{tabular}{lccccc}
\hline \multirow{2}{*}{ Cultivares } & \multicolumn{5}{c}{ Pureza (\%) } \\
\cline { 2 - 6 } & $\mathrm{C}$ & $\mathrm{E}$ & $\mathrm{ET}$ & $\mathrm{G}$ & $\mathrm{S}$ \\
\hline BRS 508 & $83.83 \mathrm{aB}$ & $82.75 \mathrm{aB}$ & $88.35 \mathrm{aA}$ & $77.78 \mathrm{bC}$ & $77.86 \mathrm{bC}$ \\
BRS 509 & $82.51 \mathrm{aAB}$ & $83.79 \mathrm{aA}$ & $80.23 \mathrm{bB}$ & $84.40 \mathrm{aA}$ & $80.83 \mathrm{aB}$ \\
\hline & DMS coluna: 1.95 & & DMS linha: 2.78 & &
\end{tabular}

Letras minúsculas comparam médias na coluna; Letras maiúsculas comparam médias na linha; Médias seguidas de letras iguais não diferem entre si, pelo teste de Tukey, a $5 \%$ de probabilidade, dentro do mesmo fator; DMS - Diferença mínima significativa; C - Controle - ausência de maturador; $\mathrm{E}$ - Etefon $\left(0,7 \mathrm{~L} \mathrm{ha} \mathrm{h}^{-1}\right) ; \mathrm{ET}$ - Etiltrinexapac $\left(0,8 \mathrm{~L} \mathrm{ha} \mathrm{h}^{-1}\right) ; \mathrm{G}-\mathrm{Glifosato}(0,4 \mathrm{~L}$ $\left.\mathrm{ha}^{-1}\right)$ e S - Sulfometurom metil $\left(0,02 \mathrm{~kg} \mathrm{ha}^{-1}\right)$. Lowercase letters compare averages in the column; Capital letters compare averages in the line; Averages followed by equal letters do not differ from each other by the Tukey test at $5 \%$ probability within the same factor; DMS - Minimum significant difference; C - Control - absence of maturer; $E$ - Ethephon (0.7 L ha $\left.{ }^{-1}\right)$; ET - Ethyltrinexapac (0.8 L ha-1); G - Glyphosate $\left(0.4 \mathrm{~L} \mathrm{ha}^{-1}\right)$ and S - Sulfometurom methyl $\left(0.02 \mathrm{~kg} \mathrm{ha}^{-1}\right)$.

Foi observada diferença significativa entre as cultivares de sorgo-sacarino para a Pureza do caldo. A cultivar BRS 508 apresentou maiores valores de pureza no tratamento com maturador químico etiltrinexapac, enquanto, para a cultivar BRS 509 , os maiores valores foram obtidos nas plantas tratadas com os maturadores glifosato e etefon (Tabela 8). Os resultados neste trabalho são semelhantes aos ocorri- 
dos em cana-de-açúcar, conforme Caputo et al. (2007), que também encontraram maiores valores de pureza após a aplicação de sulfometurom metil. Leite et al. (2009c) observaram que o sulfometurom metil e o glifosato, quando aplicados de forma isolada, elevaram de forma significativa a pureza do caldo de cana.

Sabe-se que a pureza do caldo tem correlação com o processo de maturação da cana-de-açúcar, recomendando níveis mínimos de 80 \% para o início e de $85 \%$ no decorrer da safra (Fernandes, 2011). Da mesma forma, Viana et al. (2008) também encontraram maiores valores de pureza após a aplicação de sulfometurom metil.

São apresentados na Tabela 9 os valores médios relativos ao desdobramento da interação significativa entre as duas cultivares de sorgo-sacarino submetidas à aplicação de maturadores químicos, para a característica tecnológica Fibra.

Tabela 9 - Valores médios relativos ao desdobramento da interação significativa entre as duas cultivares de sorgo-sacarino submetidas à aplicação de maturadores químicos, para a característica tecnológica Fibra, Andradina-SP, 2015. Average values related to significant interaction between the two cultivars of sweet sorghum submitted to chemical ripeners application for fiber, Andradina, São Paulo State - Brazil, 2015.

\begin{tabular}{lccccc}
\hline \multirow{2}{*}{ Cultivares } & \multicolumn{5}{c}{$\begin{array}{c}\text { Fibra } \\
(\%)\end{array}$} \\
\cline { 2 - 6 } & $\mathrm{C}$ & $\mathrm{E}$ & $\mathrm{ET}$ & $\mathrm{G}$ & $\mathrm{S}$ \\
\hline BRS 508 & $23.22 \mathrm{aA}$ & $21.32 \mathrm{aB}$ & $22.94 \mathrm{aA}$ & $20.86 \mathrm{bB}$ & $21.21 \mathrm{aB}$ \\
BRS 509 & $19.82 \mathrm{bB}$ & $19.88 \mathrm{bB}$ & $20.23 \mathrm{bB}$ & $23.55 \mathrm{aA}$ & $20.43 \mathrm{bB}$ \\
\hline & DMS coluna: 0.42 & & DMS linha: 0.61 & & \\
\hline
\end{tabular}

Letras minúsculas comparam médias na coluna; Letras maiúsculas comparam médias na linha; Médias seguidas de letras iguais não diferem entre si, pelo teste de Tukey, a $5 \%$ de probabilidade, dentro do mesmo fator; DMS - Diferença mínima significativa; $\mathrm{C}$ - Controle - ausência de maturador; $\mathrm{E}$ - Etefon $\left(0,7 \mathrm{~L} \mathrm{ha}^{-1}\right) ; \mathrm{ET}$ - Etiltrinexapac $\left(0,8 \mathrm{~L} \mathrm{ha} \mathrm{h}^{-1}\right) ; \mathrm{G}$ - Glifosato $(0,4 \mathrm{~L}$ $\left.\mathrm{ha}^{-1}\right)$ e $\mathrm{S}$ - Sulfometurom metil $\left(0,02 \mathrm{~kg} \mathrm{ha}^{-1}\right)$. Lowercase letters compare averages in the column; Capital letters compare averages in the line; Averages followed by equal letters do not differ from each other by the Tukey test at $5 \%$ probability within the same factor; DMS - Minimum significant difference; $C$ - Control - absence of maturer; $E$ - Ethephon (0.7 L ha $\left.{ }^{-1}\right)$; ET - Ethyltrinexapac (0.8 L ha-1); G - Glyphosate $\left(0.4 \mathrm{~L} \mathrm{ha}^{-1}\right)$ and S - Sulfometurom methyl $\left(0.02 \mathrm{~kg} \mathrm{ha}^{-1}\right)$.

Para a característica Fibra, houve diferença significativa entre as cultivares de sorgo-sacarino, sendo que a cultivar BRS 509 apresentou maiores valores quando tratadas com o maturador glifosato. No entanto, para a cultivar BRS 508, os tratamentos que apresentaram maiores valores para característica tecnológica Fibra foram: controle e etefon (Tabela 9). $\mathrm{O}$ aumento no teor de fibra em cana-de-açúcar, decorrente da aplicação de maturadores químicos, já foi observado por Viana et al. (2008) e Leite \& Crusciol (2008). Porém, Caputo et al. (2008) e Leite et al. (2009c) não observaram alterações significativas no teor de fibra mediante a aplicação de maturadores sem misturas.Os teores de fibra encontrados neste trabalho seguiram os resultados relatados por Silva et al. (2010), que também apontaram aumento dessa característica na cana-de-açúcar, por meio da aplicação dos fitorreguladores glifosate e sulfometuron methyl.

$\mathrm{Na}$ Tabela 10, são apresentados os valores médios relativos ao desdobramento da interação significativa entre as duas cultivares de sorgo-sacarino submetidas à aplicação de maturadores químicos, para a característica tecnológica Açúcares totais recuperáveis. Houve diferença significativa entre as cultivares de sorgo-sacarino, sendo que a cultivar BRS 509 apresentou melhores resultados quando tratadas com etiltrinexapac na característica tecnológica açúcares totais recuperáveis, enquanto, para a cultivar BRS 508, os melhores resultados foram observados quando foram aplicados os maturadores químicos etefon e sulfometurom metil. Segundo Benedini \& Ricci-Júnior (2009), o açúcar total recuperável (ATR) é importante para a indústria sucroenergética para estimar a quantidade de sacarose na matéria-prima que poderá ser recuperada como açúcar cristal. Esses resultados são semelhantes àqueles encontrados para as características tecnológicas Brix, Pol e açúcares redutores totais, que tiveram como destaque as misturas acima citadas.

Para a cultivar BRS 509, o tratamento com maturador etiltrinexapac exibiu o melhor resultado nos valores de ATR, quando comparado com os demais maturadores químicos, enquanto, para a cultivar BRS 508 , os tratamentos com maturadores químicos etefon e sulfometurom metil apresentaram melhores valores quando avaliada a característica tecnológica Açúcares totais recuperáveis (Tabela 10).

Os valores de ATR encontrados neste trabaIho são semelhantes aos relatados por Silva et al. (2010), que, em estudo sobre fitorreguladores químicos em cana-de-açúcar, aplicados em final de safra, constataram aumento significativo sobre a característica tecnológica ATR ao longo das épocas de colheitas, quando utilizado sulfometuron methyl a $20 \mathrm{~g} \mathrm{ha}^{-1}$.

Vale ressaltar que não existem, hoje, muitos trabalhos na literatura com sorgo-sacarino, com uso de maturadores químicos, por isto este estudo surge com grande importância na qualidade agroindustrial. 
Tabela 10 - Valores médios relativos ao desdobramento da interação significativa entre as duas cultivares de sorgo-sacarino submetidas à aplicação de maturadores químicos, para a característica tecnológica Açúcares totais recuperáveis, Andradina-SP, 2015. Mean values related to the significant interaction between the two sweet sorghum cultivars submitted to the application of chemical ripeners, for the technological characteristic Sugars total recoverable, Andradina, São Paulo State - Brazil, 2015.

\begin{tabular}{lccccc}
\hline \multirow{2}{*}{ Cultivares } & \multicolumn{7}{c}{$\begin{array}{c}\text { ATR } \\
(\mathrm{kg} / \mathrm{t})\end{array}$} \\
\cline { 2 - 6 } & $\mathrm{C}$ & $\mathrm{E}$ & $\mathrm{ET}$ & $\mathrm{G}$ & $\mathrm{S}$ \\
\hline BRS 508 & $135.59 \mathrm{aC}$ & $144.35 \mathrm{aA}$ & $138.29 \mathrm{bB}$ & $128.98 \mathrm{aD}$ & $143.89 \mathrm{aA}$ \\
BRS 509 & $136.36 \mathrm{aC}$ & $144.76 \mathrm{aB}$ & $149.30 \mathrm{aA}$ & $126.39 \mathrm{bD}$ & $137.56 \mathrm{bC}$ \\
\hline \multicolumn{7}{c}{ DMS coluna: 0.77} & & DMS linha: 2.53 & & \\
\hline
\end{tabular}

Letras minúsculas comparam médias na coluna; Letras maiúsculas comparam médias na linha; Médias seguidas de letras iguais não diferem entre si, pelo teste de Tukey, a 5\% de probabilidade, dentro do mesmo fator; DMS - Diferença mínima significativa; C - Controle - ausência de maturador; E - Etefon $\left(0,7 \mathrm{~L} \mathrm{ha}^{-1}\right) ; \mathrm{ET}$ - Etiltrinexapac $\left(0,8 \mathrm{~L} \mathrm{ha}^{-1}\right) ; \mathrm{G}-\mathrm{Glifosato}(0,4 \mathrm{~L}$ $\left.\mathrm{ha}^{-1}\right)$ e S - Sulfometurom metil $\left(0,02 \mathrm{~kg} \mathrm{ha}^{-1}\right)$. Lowercase letters compare averages in the column; Capital letters compare averages in the line; Averages followed by equal letters do not differ from each other by the Tukey test at $5 \%$ probability within the same factor; DMS - Minimum significant difference; $C$ - Control - absence of maturer; $E$ - Ethephon (0.7 L ha $\left.{ }^{-1}\right)$; ET - Ethyl-

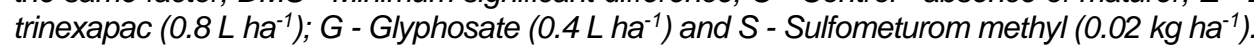

\section{Conclusões}

De maneira geral, a aplicação de maturadores químicos na cultura do sorgo-sacarino proporcionou aumento das características tecnológicas. A cultivar BRS 508 apresentou aumento na qualidade tecnológica quando utilizado etefon $\left(0,7 \mathrm{~L} \mathrm{ha}^{-1}\right)$ e sulfometurom metil $\left(0,02 \mathrm{~kg} \mathrm{ha}^{-1}\right)$ como maturadores químicos. A cultivar BRS 509 apresentou ganhos na qualidade tecnológica quando aplicado o etiltrinexapac $\left(0,8 \mathrm{~L} \mathrm{ha}^{-1}\right)$ como maturador químico.

\section{Referências}

Almodares A, Hadi MR (2009) Production of bioethanol from sweet sorghum: a review. African Journal of Agricultural Research 4(9):772-780.

Benedini MS, Ricci-Júnior AR (2009) Respostas das variedades CTC a maturadores. Revista Coplana 56:30-32.

Caputo MM, Beauclair EGF, Silva MA, Piedade SMSP (2008) Resposta de genótipos de cana-de-açúcar à aplicação de indutores de maturação. Bragantia 67: 15-23.

Caputo MM, Silva MA, Beauclair EGF, Gaya GJC (2007) Acúmulo de sacarose, produtividade e florescimento de cana-de-açúcar sob reguladores vegetais. Interciência 32(12):834-840.

Consecana - Conselho dos Produtores de Cana-deaçúcar, Açúcar, Álcool do Estado de São Paulo (2006) Manual de instruções. Consecana. 112p.

Durães FOM (2011) Sorgo sacarino: desenvolvimento de tecnologia agronômica. Revista em Agroenergia 3:7.

Fernandes AC (2011) Cálculos na agroindústria de cana-de-açúcar. STAB, Açúcar, Álcool e Subprodutos. $416 p$.
Gomide ALO, Veloso LA, Lavanholi MGP (2008) Uso dos maturadores químicos na cultura da cana-de-açúcar (Saccharum spp.). Nucleus Edição Especial, 19-27.

Leite GHP, Crusciol CAC, Silva MA, Venturini Filho WG (2009a) Qualidade tecnológica da cana-de-açúcar em função da aplicação de maturadores em meio de safra. Bragantia 68:527-534.

Leite GHP, Crusciol CAC (2008). Reguladores vegetais no desenvolvimento e produtividade da cana-deaçúcar. Pesquisa Agropecuária Brasileira 43(8): 9951001.

Leite GHP, Crusciol, CAC, Lima GPP, Silva MA (2009c) Reguladores vegetais e atividade de invertases em cana-de-açúcar em meio de safra. Ciência Rural 39:718-725.

Leite GHP, Crusciol, CAC, Silva MA (2011) Desenvolvimento e produtividade da cana-de-açúcar após aplicação de reguladores vegetais em meio de safra. Semina Ciências Agrárias 32:129-138.

May A, Albuquerque Filho MR, Rodrigues JAS, Landau EC, Parrella RAC, Massafera R. (2011). Cultivares de sorgo para o mercado brasileiro na safra 2011/2012. Empresa Brasileira de Pesquisa Agropecuária. $28 \mathrm{p}$.

Meschede DK, Velini ED, Carbonari CA, Silva JRM (2011) Physiological alteration in sugarcane influenced by glyphosate and sulfumeturon-methyl application. Planta Daninha 29(2):413-419.

Muhwiridzwa M, Manenji BT, Madanzi T, Mahohoma W (2016). Evaluating the effects of chemical ripening with fluazifop-p-butyl on sugarcane (Saccharum officinarum) yield and sugar content. International Journal of Plant \& Soil Science 9(6):1-8.

Prasad S, Singh A, Jain N, Joshi HC (2007) Ethanol production from sweet sorghum syrup for utilization as automotive fuel in India. Energy and Fuels, 21:2415-2420. 
Raij B \& Cantarella H (1996) Outras culturas industriais. In: Raij B, Cantarella H, Quaggio JA, Furlani AMC (ed) Recomendações de adubação e calagem para o Estado de São Paulo, Instituto Agronômico e Fundação IAC. p.233-236.

Sipos B, Réczey J, Somorai Z, Kádár Z, Dienes D, Réczey K (2009) Sweet sorghum as feedstock for ethanol production: enzymatic hydrolysis of steampretreated bagasse. Applied Biochemistry and Biotechnology 153:151-62.

Silva MA, Gava, GJC, Caputo MM, Pincelli RP, Jerônimo EM, Cruz JCS (2007) Uso de reguladores de crescimento como potencializadores do perfilhamento e da produtividade em cana-soca. Bragantia 66(4):545-552.

Silva FAZ \& Azevedo CAV (2002). Versão do programa computacional Assistat para o sistema operacional Windows. Revista Brasileira de Produtos Agroindustriais, Campina Grande, 4(1):71-78, 2002.

Silva MA, Gava GJC, Caputo MM, Sorrila PF, Bassetto SC (2010) Produtividade de colmos e de sacarose em cana-de-açúcar em função da interação entre cultivares e maturadores. Scientia Agraria 11(4): 323-329.
Viana RS, Silva PH, Mutton MA, Mutton MJR, Guimarães ER, Bento M (2008) Efeito da aplicação de maturadores químicos na cultura da cana de açúcar (Saccharum spp.) variedade SP 81-3250. Acta Science Agronomy 30(1): 65-71.

Viana RS, Figueiredo PAM, Lisboa LAM, Assumpção ACND, Sá ME, May A (2016). Aplicação de fitorreguladores químicos na qualidade tecnológica do sorgo sacarino cv. Biomatrix 535. Revista Brasileira de Milho e Sorgo 14(3):326-334.

Viana RS, Figueiredo PAM, Lisboa LAM, Magalhães AC (2016). Morpho-anatomical aspects and technological quality of saccharine sorghum submitted to plant regulators. Revista Brasileira de Herbicidas 15(2):175-183.

Viana RS, Mutton MA, Zillo H (2015). Índices de maturação da cana-de-açúcar quando submetida à aplicação de maturadores químicos. Revista Mirante 8(1):99-109. 